\title{
UNA RICERCA DELLO STILE: LE PRIME RACCOLTE IN DIALETTO TRIESTINO DI CAROLUS LUIGI CERGOLY
}

\author{
Eliana Moscarda Mirković \\ Università Juraj Dobrila di Pola \\ Dipartimento di studi in lingua italiana
}

\section{Riassunto}

L'indagine di questo saggio si concentra sulle prime raccolte di versi triestini di Carolus L. Cergoly, Dentro de mi e Poesie a Barbara. Attraverso una scelta di liriche rappresentative si definisce l'impianto strutturale di queste prime sillogi, con esempi stilistici che permettono di definire il linguaggio poetico cergolyano. Il linguaggio che emerge è un modo di fare poesia che segue schemi inconsueti, perché mentre svela il funzionamento della lingua, provoca accostamenti inediti. Tra gli espedienti tecnici si nota soprattutto una parola ampliata in un'utilizzazione sintattica che ne rende instabile anche il ruolo. La decostruzione e la costruzione valgono, pertanto, sia come operazioni strumentali di una negazione della parola univoca e monovalente, sia come realizzazione di un linguaggio calato nel modo di montare il materiale linguistico.

Parole chiave: Cergoly, Trieste, poesia, dialetto.

Carolus L. Cergoly, in riferimento alla sua fase poetica in dialetto triestino, è il primo a parlare di scelta di un lessico ${ }^{1}$ e non di un dialetto. Anche se per il dialetto cergolyano non è lecito parlare di lingua, possiamo certamente definirlo un linguaggio dalle forme aperte in cui possono sommarsi contributi di ogni genere, dalle parole straniere a quelle di antichi lessici letterari, dai neologismi agli hapax legomena.

La prima raccolta di versi triestini è Dentro de mi (1938), la seconda è Poesie a Barbara (1943) con soli quattro componimenti con una tiratura di cinquanta esemplari. Le liriche di queste due sillogi entreranno, con altre due poesie, nel volume Il portolano di Carolus. Poesie in dialetto triestino (1970). Come nota Damiani ${ }^{2}$, si tratta di componimenti distaccati dalla lezione vernacolare e da ogni influenza giottiana

1 A tal proposito basti notare i titoli delle sillogi successive:

Cergoly C. L.: Canti clandestini. Nove poesie in lessico triestino, Trieste, Tullio Misan libraio-editore, 1972; Hohò Trieste. Ballatetta in lessico triestino, Trieste, Editrice La Galleria dei Rettori, 1973; Ponterosso. Poesie mitteleuropee in lessico triestino, «Quaderni della Fenice» n. 10, Parma, Guanda, 1976; Latitudine nord. Tutte le poesie mitteleuropee in lessico triestino, Milano, Mondadori, 1980.

La definizione «lessico triestino» inizia ad essere usata da Cergoly dagli anni ‘70 in poi. Ma Roberto Damiani ritiene che il termine «lessico» sia funzionale alla presa di distanza dalla locale «scuola vernacolare» e che tale definizione sia però inappropriata da un punto di vista scientifico. Cfr. Damiani R., Poeti dialettali triestini. Profilo storico-critico (18751980), Trieste, Lafanicola Edizioni «Italo Svevo», 1981, p. 138.

2 Damiani R.- Grisancich C. (a cura di), La poesia in dialetto a Trieste, Trieste, Edizioni «Italo Svevo», 1989 , p. 26. 
(per ragioni cronologiche ma anche per una questione di gusto e di educazione ${ }^{3}$ ), e la loro peculiarità risiede nell'utilizzo di semplificazioni formali di diretta ascendenza futurista e nell'innesto sul ceppo dell'avvenuta semplificazione di un sentimento onesto di amabile affabulatore.

Nessun intento mimetico di riproduzione di realtà extra-linguistiche, nessun tentativo di innalzarsi miticamente a interprete del popolo, scendendo a livello di questo, caratterizza la poesia di Cergoly ${ }^{4}$. Il poeta sa di scrivere in una lingua non certo provinciale, bensì ricca di valenze espressive e perciò partecipe di un sistema linguistico più aperto. La sua direzione, infatti, non è, come accade alla maggior parte dei poeti in dialetto (e dei triestini che l'hanno preceduto), da una lingua maggiore ad una minore, dalla lingua nazionale al linguaggio del popolo triestino ${ }^{5}$. Bensì, come afferma Pier Paolo Pasolini nel suo saggio Carolus L. Cergoly, Inter pocula, poesie segrete triestine:

Nel caso che ho oggi sul tavolo del laboratorio [...] il modo di essere «poeta dialettale» scelto dall'autore è ancora un modo nuovo rispetto a tutti quelli che io possa aver sperimentato nella mia ormai lunga frequentazione di testi dialettali.

Carolus L. Cergoly infatti adopera il dialetto triestino come se esso non fosse affatto lo strumento comunicativo usato dalle classi subalterne, cioè della piccola e infima borghesia e dal popolo. [...] Cergoly usa il triestino come una qualsiasi persona colta e ricca di Trieste all'interno del proprio mondo classista. Ma il suo modo di usare sulla pagina il triestino è poi perfettamente uguale a quello in cui egli usa il triestino parlando: lo scrive cioè da persona colta e ricca, esattamente come lo parla ${ }^{6}$.

Il primo approccio alla poesia, ascrivibile alla fase giovanile della produzione poetica di Cergoly, è tendenzialmente acritico e sentimentale: un rifugio nella sfera della sensibilità individuale privata, dominata da un'alienazione estetica e sentimentale, per cui il poeta dà una visione impressionistica della realtà, ammantata di un fascino penetrante e, di conseguenza, dispersa, nel frammento ${ }^{7}$. Un frammento nel quale si rispecchia la tormentata ricerca di una propria definizione, quel punto fermo della personalità che accomuna tematicamente tutta la letteratura triestina del Novecento ${ }^{8}$.

3 Damiani R., Oggi, i poeti in dialetto, in «La Battana» A. X n. 30-31, Fiume, Edit, 1973, p. 85.

4 Borsetto L., Trieste segreta. Le poesie mitteleuropee di Carolus L. Cergoly, in «La Rassegna della letteratura italiana», Anno $82^{\circ}$, n. 12, Firenze, Sansoni, 1978, p. 194.

5 È la direzione seguita da Virgilio Giotti, che dal linguaggio del popolo ha tratto un proprio linguaggio, come tutti i grandi dialettali del Novecento, i quali dalla lingua della realtà hanno tratto la lingua della poesia. Cfr. CHIESA M.-TeSIO G. (a cura di), Il dialetto da lingua della realtà a lingua della poesia, Torino, Paravia, 1978.

6 Pasolini P. P., Carolus L. Cergoly, Inter pocula, poesie segrete triestine, in Saggi sulla letteratura e sull'arte, Tomo secondo (a cura di Siti W.-De Laude S.), Milano, Mondadori, 1999, pp. 2108-2109.

7 Così Claudio Magris definisce i caratteri che contraddistinguono la letteratura austriaca moderna che tende a rappresentare il clima della decadenza asburgica in termini impressionistici e frammentari: «È una vera e propria fuga nel frammento, nel particolare; un evadere dai problemi nella leggerezza di un'ora, di un gesto, di un sorriso. La realtà viene per così dire frantumata, e ne rimangono deliziosi brani avulsi dal contesto, immagini delicate e subito svanenti come bolle di sapone. La civiltà asburgica era sempre rifuggita da una visione unitaria della vita, dalla percezione dei concreti rapporti storici tra gli eventi, per cui i diversi aspetti del reale si configuravano come tante linee parallele, ognuna per sé stante. [...] Si tratta di un'alienazione estetica e sentimentale, per cui ci si rifugia nella sfera della sensibilità individuale e si ammanta la realtà di un fascino capriccioso e pungente». Cfr. MAGRIS C., Il mito absburgico nella letteratura austriaca moderna, Torino, Einaudi, 2009, p. 204.

8 «Questi scrittori di lingua, di cultura e spesso di sangue misto, sono spesso intenti a scoprirsi, a definirsi, a cercare il loro punto fermo; ma quasi col presupposto di non trovarlo; come chi faccia della ricerca non il mezzo, ma addirittura il fine del suo cercare». PAncrazi G., Scrittori d'oggi, Bari, Laterza, Serie seconda, 1946, p. 304. 
Le raccolte Dentro de mi e Poesie a Barbara confluiscono integralmente nell'edizione de Il Portolano, mentre nella successiva Latitudine nord Cergoly omette le seguenti liriche: Xe rivà i paiassi, Vestidi de tarlis per la via, Con lu andavo, Drio $i$ nuvoli se scondi i nostri morti ${ }^{9}$. Ciò che si nota nell'edizione del 1980, è che le poesie sono sottratte ai primitivi raggruppamenti e subiscono variazioni anche nella stesura, con un allineamento posizionale diverso rispetto alla prima edizione. Ne emerge la volontà cergolyana di accentuare il carattere di un'operazione in fieri, aperta a nuovi esiti e disposta, fino all'ultimo, a rinnovarsi e rimettersi in gioco. Un gioco di strutture compositive che si ripercuote in forme metriche aperte, che affrontano tematicamente motivi cosiddetti eterni, che ritorneranno in molteplici variazioni nelle sillogi successive. Quello cergolyano è un parlare intimistico di affettività nelle sue diverse intensità e sfumature (con un mormorio di voci della quotidianità percepito come costante), che trova il suo movimento nella freschezza di un paesaggio fatto soprattutto di onde marine, di volo di gabbiani e di «grembani» del Carso.

In Latitudine nord scompaiono innanzitutto anche quei pochi segni d'interpunzione che Cergoly aveva inserito nella prima stesura e presenti in Il portolano. A titolo esemplificativo si veda la lirica El mio amor Amor ${ }^{10}$.

El mio amor Amor
Come se ciama?
Lydia, Dorina, Gemma
Gocia de mar o Carso
Fureto o cingalegra
Salvia, somaco, menta?

Tuto insolà rispondi

Amor: fior la ga nome

Fato de piera

Piantà su creste

D'onde marine

La se ciama Trieste ${ }^{11}$

La se ciama Trieste ${ }^{12}$
El mio amor Amor

Come se ciama

Lydia Dorina Gemma

Goccia de mar o Carso

Furreto o cingallegra

Salvia sommaco menta

Tutto insolà rispondi

Amor

Fior la ga nome

Fatto de piera

Piantà su creste

D'onde marine

Poeticamente «creste» e «piera» sono i tratti, a indagarli nella loro scabra essenza, del «ragazzaccio aspro e vorace» amato da Saba. Soluzioni artistiche a parte, si ravvisa sia in Cergoly sia in Saba la medesima esigenza di purezza, la stessa necessità

9 Le liriche di Cergoly in dialetto triestino non presentano titoli. Pertanto, nel corso del lavoro, nel riferimento alle singole poesie, si riporteranno i primi versi. Altra particolarità di questi versi è che ogni parola ad inizio di verso è scritta con la lettera maiuscola.

10 Due sono le liriche cergolyane che hanno lo stesso incipit: quella sopra riportata e un'altra inserita in Latitudine nord. Tutte le poesie mitteleuropee in lessico triestino, Milano, Mondadori, 1980, p. 85.

11 Cergoly C. L., El mio amor Amor, in Il portolano di Carolus. Poesie in dialetto triestino, cit., p. 1.

«Il mio amore Amore / Come si chiama? / Lydia, Dorina, Gemma / Goccia di mare o Carso / Furetto o cinciallegra / Salvia, sommacco, menta? // Tutto pieno di sole risponde / Amore: fiore si chiama / Fatto di pietra / Piantato su creste / Di onde marine / Si chiama Trieste».

12 Id., El mio amor Amor, in Latitudine nord. Tutte le poesie mitteleuropee in lessico triestino, cit., p. 15. 
radicale di smitizzare con la parola ciò che il sentimento ama con certezza, proprio in quanto smitizzato da ogni tentazione coloristica o «letteraria» ${ }^{13}$.

Nella lirica sopra riportata, stilisticamente va notato l'uso del poliptoto nel primo verso, ovvero la doppia funzione di «amor», con valenza di soggetto (con l'iniziale in minuscolo) e di vocativo (con l'iniziale in maiuscolo). Tanto che l'oggetto dell'enunciazione «Trieste» si sposta dal soggetto dialogico della prima strofa corrispondente al poeta, a quello della seconda «Amor».

Mettendo a confronto le due edizioni, salta subito all'occhio come il punto interrogativo scompaia dal secondo e sesto verso, le virgole vengano omesse dalla terna nominale del terzo e sesto verso e i due punti non introducano più il discorso diretto, che dal secondo verso della seconda strofa nell'edizione de Il Portolano slitta al terzo verso nell'edizione di Latitudine nord (e la struttura complessiva della lirica ritmicamente priva di sussulti, si amplia di un verso rispetto alla precedente edizione, portando il numero di versi a tredici). Sempre dal lato grammaticale, in corsivo si sono voluti evidenziare gli ipercorrettismi di più stretta aderenza alla koiné italiana, che Cergoly inserisce nella seconda edizione, rispetto alla valorizzazione del triestino parlato della precedente, che non prevede l'uso delle consonanti doppie (fureto / furetto, cingalegra / cingallegra, fato / fatto, somaco / sommaco $^{14}$ ).

Questa della sovrabbondanza consonantica è una scelta che caratterizza sia la raccolta Ponterosso sia Latitudine nord, che in alcuni casi presentano vistose differenze grafiche rispetto alla prima stesura. Qui di seguito si riportano solo alcuni esempi (segnalati in corsivo):

Scolto sbrissar un'onda ${ }^{15}$ - Scolto $a$ sbrissar un' onda ${ }^{16}$

Gavemo granda l'anima / Gonfia de amor ${ }^{17}$ - Gavemo grande l'anima / Gonfia d'amor ${ }^{18}$

$\mathrm{Su}$ le piere lisse ${ }^{19} / \mathrm{Su}$ le piere liscie $^{20}$

Cergoly ricorre spesso anche alla tendenza di stravolgere l'ordine non marcato della frase attraverso l'inversione supportata dall'uso di enjambement:

[...]

Che povero zerca el passo

Tuo per caminar pulito

E la tua forza ch'el sasso

13 Così Damiani R. in Oggi, i poeti in dialetto, cit., p. 60.

14 Ma per sommaco Cergoly non rispetta completamente la corretta grafia, tralasciando l'aggiunta di una $c$.

15 Cergoly C. L., Mio fradel e mi, in Il portolano di Carolus, cit., p. 5.

16 Id., Mio fradel e mi, in Latitudine nord., cit., p. 19.

17 Id., Sora de ti i tu rizi, in Il portolano di Carolus, cit., p. 14.

18 Id., Sora de ti i tu rizzi, in Latitudine nord., cit., p. 23.

19 Id., Mio fradel e mi, in Il portolano di Carolus, cit., p. 5.

20 Id., Mio fradel e mi, in Latitudine nord., cit., p. 19. 
Trasformava in bona piera

Dai vardime mama un poco

Go sol, luna, ciaro, sera? [... $]^{21}$

con un'accumulazione di termini antitetici «sol, luna, ciaro, sera», in questa che vuole essere intimisticamente la ricerca, nella figura materna scomparsa, di un punto di riferimento esistenziale.

Anche in questo caso la lirica subisce mutamenti in Latitudine nord ${ }^{22}$. A partire dalla diversa costruzione strofica «Che povero zerca el passo / Tuo per camminar pulito / E la tua forza ch'el sasso / Trasformava in bona piera // Dai vardime mamma un poco / Go sol luna ciaro sera / No capisso dentro de mi //), vi appaiono nuovamente gli ipercorrettismi e l'assenza di punteggiatura.

In Ela dentro de $m i^{23}$, nell'edizione di Latitudine nord Cergoly rimuove completamente la seguente strofa rispetto a Il Portolano: «Se fa dei progeti / Pici ma tanto bei / De sposarse. I leti / Xe manca le cheche//». E la parola «do» con l'ennesimo ipercorrettismo diventa «due».

La poesia di Cergoly, già in questa raccolta, addotta soluzioni linguistiche che realizzano il loro movimento con un'agile tecnica espressionistica. Il linguaggio poetico che emerge è un modo di fare poesia secondo schemi inconsueti, perché mentre svela il funzionamento della lingua, provoca accostamenti inediti. La decostruzione e la costruzione valgono sia come operazioni strumentali di una negazione della parola univoca e monovalente, sia come realizzazione di un linguaggio calato nel modo di montare il materiale linguistico. Così affiora un triestino atipico, rotto da citazioni sentimentali di termini sloveni (spesso racchiusi nel virgolettato, es. «doberdan») che si fondono con termini provenienti dal favoloso mondo dell'infanzia carsica («skrat») ${ }^{24}$.

Se camina pel Carso lassando

Ciodi sui grembani. Verde se zerca

E de star soli e in pase. Passando

Se senti zighi. Usei che missia in graia

Gambe. Dentro xe tutto un matez

E par ch'el «skrat» se scondi drio ogni piera

Zigando «doberdan». Un cocolez

De vento ne la schena. Si «doberdan»

Se svolta. Tre putele coi cavei

Panocia vendi mazi de primole

21 Cergoly C. L., Mama, no te camini più, in Il portolano di Carolus. Poesie in dialetto triestino, cit., p. 2.

«[...] Che povero cerca il passo / Tuo per camminare pulito / E la tua forza che il sasso // Trasformava in buona pietra /

Dai guardami mamma un poco / Ho sole luna chiaro sera? [...]».

22 Id., Mamma no te cammini più, in Latitudine nord., cit., pp. 15-16.

23 Id., Il portolano di Carolus, cit., p. 3.

«Si fanno dei progetti / Piccoli ma tanto belli / Di sposarci. I letti / Ci sono mancano i soldi».

24 Cfr. Borsetto L., Lingua dialetto poesia. Cinque analisi: Marin, Cergoly, Calzavara, Giacomini, Bartolini, Bartolini, Ravenna, Edizioni Essegi, 1989, p. 45. 
Tre soldi. Ne le graie fis'cia usei

In fondo un frasco fa slongar le gambe ${ }^{25}$

La mancanza di nessi di relazione in cavei/panocia e in primole/tre soldi porta il sostantivo, grazie anche all'enjambement, a trasgredire alla norma assegnata alla propria categoria grammaticale e a sconfinare in quella dell'aggettivo, sostituendolo. Evidente è poi la disposizione a chiasmo dei versi quattro e undici «Ne le graie fis'cia usei»e «Usei che missia in graia».

Anchela lirica che aprelaraccolta Poesiea Barbara, composta complessivamente da cinque strofe di cui tre quartine e due distici, è tutta giocata sull'anafora con uno spessore e un volume della parola che si colloca nelle forme semplici del ritornare insistente di un suono con accezione cantilenante (prima e quinta strofa) e con costruzioni a chiasmo e anadiplosi nei distici (con anadiplosi anche tra la prima e seconda strofa).

Chi no capissi el mar no te capissi

Qua e la svola cocai

Chi no capissi el mar no te capissi

Se semo inamorai

Se semo inamorai ma come e quando

Ma quando e come se semo inamorai?

El mar xe tuto rizi imborezadi

Per le sue rive s'andemo a remenar

Tignindose per man oci nei oci

Se coniuga de dentro el verbo amar

Se semo inamorai fazendo cossa

Fazendo cossa se semo inamorai?

Lassa coi tu cavei che fazo togne

Lassa po' che le buto in questo mar

Quel che mi ciaparò faremo in due

Cùcite Barbara e vardime pescar. ${ }^{26}$

25 Cergoly C. L., Se camina pel Carso lassando, in Il portolano di Carolus, cit., p. 7.

«Si cammina per il Carso lasciando / Chiodi sui sassi spigolosi. Verde si cerca / E di stare da soli e in pace. Passando / Si sentono urla. Uccelli che mescolano nei cespugli // Gambe. Dentro è tutto un'allegra follia / E sembra che lo «skrat» si nasconda dietro ad ogni pietra / Urlando «doberdan». Una coccola / Di vento nella schiena. Sì «doberdan». // Si svolta. Tre ragazze con i capelli / Pannocchia vendono mazzi di primule / Tre soldi. Nei cespugli fischiano uccelli / In fondo una frasca fa allungare le gambe».

Lo «Škrat» è nella mitologia slovena un nano, protagonista di molti racconti e fiabe. La derivazione etimologica probabilmente risale all'antico indoeuropeo «scrat(i)», bosco dei demoni. Cergoly, forse anche per ragioni tipografiche, tralascia la corretta grafia sostituendo la consonante š con la lettera $s$.

«Doberdan» in lingua slovena significa buongiorno.

Da notare che nell'edizione della raccolta Latitudine nord Cergoly apporta due modifiche a questi versi, scrivendo «Skrat» con la lettera maiuscola (dando al termine una designazione di nome proprio) e «coccolez» raddoppiando la consonante $\mathrm{c}$ (con più aderenza all'italiano standard).

26 Cergoly C. L., Chi no capissi el mar no te capissi, in Il portolano di Carolus, cit., p. 12.

«Chi non comprende il mare non ti comprende / Qua e là volano gabbiani / Chi non comprende il mare non ti comprende 
La realtà del paesaggio marino (inserito in una struttura che rimanda ai componimenti popolari, in cui il linguaggio volutamente prosastico prende vita in una leggerezza epigrammatica) è trasfigurata nel rapporto fra il poeta e la donna amata che appare circa a metà del componimento. Vi è descritta una tipica passeggiata fra due innamorati lungo la riva triestina, in cui il mare battuto dalla bora viene osservato con divertita partecipazione dai due innamorati. Ma Barbara, in questi quattro componimenti, oltre ai dati storici che ne fissano i tratti, non sopravvive alla poesia con la stessa esplosiva passionalità ed eroticità, ad esempio, di una Bettina o di una Claribel del successivo $I l$ portolano di Carolus. E anche se personaggio autonomo, diventa «meccanismo di un ideale poetico intessuto di scarno misticismo e cartina tornasole, "velame", del vero risultato cui tende Cergoly, comunicare il suo amore incondizionato per Trieste» ${ }^{27}$.

Non si esauriscono qua gli espedienti poetici di cui fa uso Cergoly. Ed ecco apparire, nell'ambito delle due prime raccolte giovanili, altri esempi di alcune di quelle che si pongono come cifre caratterizzanti di tutta l'operazione artistica cergolyana:

- voci onomatopeiche:

crac, patatrac, cip cip, si si

- forestierismi:

bonbon ${ }^{28}$, bonzo ${ }^{29}$, libe ${ }^{30}$

- parole composte:

rampigamuri ${ }^{31}$

Ventisettembre ${ }^{32}$

\footnotetext{
/ Ci siamo innamorati // Ci siamo innamorati ma come e quando / Ma quando e come ci siamo innamorati? // Il mare è tutto ricci increspati dalla bora / Per le sue rive andiamo a gironzolare / Tenendoci per mano occhi negli occhi / Si coniuga dall'interno il verbo amare // Ci siamo innamorati facendo che cosa / Facendo che cosa ci siamo innamorati? // Lascia che con i tuoi capelli faccia lenze / Lascia poi che le butti in questo mare / Quello che prenderò lo divideremo in due / Accovàcciati Barbara e guardami pescare».

27 Damiani R., La miccia verde. Saggi di critica letteraria, Trieste, LINT, 1974, p. 65.

28 Cfr. Devoto G.-Oli G.C., Vocabolario della lingua italiana 2008, Milano, Le Monnier, 2007, p. 348: col significato di «chicca, zuccherino di pasta morbida» deriva dal francese e significa propriamente «buono-buono». È voce infantile riferita a ogni dolciume.

29 Col significato di «monaco e prete buddista» deriva dal portoghese bonzo a sua volta dal giapponese bonsō «superiore del monastero» (dal cinese fan seng «persona religiosa, prete»). Nel significato di vecchio ridicolo o rimbambito è comune anche al francese bonze (1570). Cfr. BATTISTI C.-Alessio G., Dizionario etimologico italiano, Vol. 1, Firenze, G. Barbera Editore, 1968, p. 560.

Lo zio Bonzo è anche uno dei personaggi della Madama Butterfly di Giacomo Puccini (1903).

30 Cergoly C. L., Mio fradel e mi, in Il portolano di Carolus, cit., p. 5.

Così in Pinguentini G., Nuovo dizionario del dialetto triestino. Storico, etimologico, fraseologico, Modena, Del Bianco, 1986, p. 181: «liba o tordo» pesce marino della famiglia dei Labridi: «Labrus mixtus». Vive lungo la costa dalmata ed istriana e si distingue per il differente colorito dei maschi e delle femmine. I primi sono gialli con striature azzurre, le seconde rosee, con tre macchie nere sul dorso; in Doria M., Grande dizionario del dialetto triestino. Storico, etimologico, fraseologico, Trieste, Edizione «Trieste Oggi», 1991, p. 329: «liba» sostantivo femminile, ittiologico, crenilabro; voce d'origine croata.

Riteniamo di non sbagliare affermando che in Cergoly il termine sia un iperonimo e assuma un significato più ampio rispetto a quello dell'iponimo indicato dai dizionari, andando a riprendere la voce croata «ribe» col significato di pesci. Le «bone libe» della lirica Mio fradel e mi non ci sembra altro che la trasposizione del linguaggio infantile in cui, per la difficoltà di pronuncia della consonante alveolare sonora vibrante $r$, questa viene resa con l'alveolare sonora laterale $l$.

31 Cergoly C. L., Vestidi de tarlis per la via, in Il portolano di Carolus, cit., p. 9.

32 Id., Sentà. La sigaretta, ivi, p. 25.
} 
- sineddoche:

Vestida a fiori ${ }^{33}$

- enumerazione per asindeto:

Sul mar sui monti in bosco;

Per la bora la piera el mar ${ }^{34}$

I se distira, i canta i cori i zoga / I ne varda i ne parla (con anafora) ${ }^{35}$

- e polisindeto:

Albero e piera nuvolo e mar ${ }^{36}$

- costruzione ad iperbato che ritarda l'apparizione del soggetto su cui poggia la tensione del discorso:

Paraduro de mar / Al mio viver mato / Te ieri ti ${ }^{37}$

- frasi da insegna:

La tabella disi: Spiriti e Vin ${ }^{38}$

- frasi di taglio giornalistico:

«El Lloyd in borsa crac» ${ }^{39}$ (che diventa crak in Latitudine nord ${ }^{40}$, con doppia funzione di forestierismo e voce onomatopeica);

- insistenza sul colore verde con diverse funzioni grammaticali (soggettivale, oggettivale ed avverbiale):

verde se zerca ${ }^{41}$ (con uso dell'inversione)

maciade de verde ${ }^{42}$

Magari poderse vestir / Sempre de verde ${ }^{43}$

se camina / vestidi de verde ${ }^{44}$

Mi vento che te cocola / Ti verde che se lassa dondolar ${ }^{45}$

I verdi ghe piaseva ${ }^{46}$

- perifrasi ottenute dall'incontro di morfemi modificanti:

prefisso in + aggettivo

E caminar per stradoni / Inzalai de sol ${ }^{47}$

prefisso in + sostantivo

Tuto insolà rispondi ${ }^{48}$.

33 Id., Xe rivà i paiassi, in Il portolano di Carolus, cit., p. 4.

34 Id., Sora de ti i tu rizi, in Il portolano di Carolus, cit., p. 14.

35 Id., Drio nuvoli se scondi i nostri morti, in Il portolano di Carolus, cit., p. 13.

36 Cergoly C. L., Butada in mar la piera, in Il portolano di Carolus, cit., p. 15.

37 Id., Paraduro de mar, in Il portolano di Carolus, cit., p. 16.

38 Id., Vestidi de tarlis per la via in Il portolano di Carolus, cit., p. 9.

39 Id., Se sua mama vedessi, ivi, p. 6.

40 Id., Se sua mama vedessi, in Latitudine nord, cit., p. 17.

41 Id., Se camina pel Carso lassando, in Il portolano di Carolus, cit., p. 7.

42 Id., Su la schena de mar no xe vapori, ivi, p. 19.

43 Id., Magari poderse vestir, ivi, p. 11.

44 Ivi, p. 11.

45 Id., Butada in mar la piera, ivi, p. 15.

46 Id., Con lu andavo, ivi, p. 10.

47 Cergoly C. L., Magari poderse vestir, ivi, p. 11.

48 Id., El mio amor Amor, ivi, p. 1. 
Nell'atmosfera poi dei seguenti versi «[...] Due giorni forsi. Po ziga / Zivete e svola / Pipistrei torno la testa / Ongie se magna /[...] / Dopo se vedi pipistrei / Zivete morte $49 /[\ldots] »$, ci sembra di poter cogliere un'intertestualità a livello formale dello Spleen (1857) di Charles Baudelaire «[...] / Quand le terre est changée en un cachot humide, / Où l'Espérance, comme une chauve-souris, / S'en va battant les murs de son aile timide / Et se cognant la tête à des plafonds porri. //» ${ }^{50}$. Per l'immagine epigrammatica di «[...] Come questo balon / Scampà de man / In piazza a la putela ${ }^{51}$ si può ritenere che Cergoly potesse avere come punto di riferimento anche i versi pascoliani de L'Aquilone: [...] Ed ecco ondeggia, pencola, urta, sbalza, / risale, prende il vento; ecco pian piano / tra un lungo dei fanciulli urlo s'innalza. // S'innalza; e ruba il filo dalla mano, / come un fiore che fugga su lo stelo / esile, e vada a rifiorir lontano. // S'innalza; e i piedi trepidi e l'anelo / petto del bimbo e l'avida pupilla / e il viso e il cuore, porta tutto in cielo. / [...] Come si è potuto notare, nel caso di Cergoly la poesia degli anni Trenta e Quaranta dello scorso secolo si basa su di un «realismo» che muove da forme di diversa misura, mimetiche della quotidianità triestina, ma anche degli ambienti periferici e provinciali del Carso. È una poesia in cui domina la celebrazione dell'immediatezza del vissuto. La raccolta Dentro de mi diventa un insieme di quadri e di vicende, in cui figura la rappresentazione di Trieste con i suoi luoghi d'incontro e la molteplicità di esistenze che vi si incrociano, ma che rappresentano soprattutto le stagioni della vita del poeta e delle sue vicende sentimentali. In questi versi convivono armonicamente l'idea della poesia come gioco e la spontaneità esistenziale fatta di brulicanti esperienze vitali. Un «realismo» trasmesso dalle cose alle strutture linguistiche, descrittivo pur se «straniato», che si realizza immediatamente a livello formale nel tentativo di fissare la parola in dimensioni materiche, di farla risuonare nella ripresa anaforica. La derivazione da aree referenziali tematicamente e ideologicamente ben definite, predispone il materiale linguistico a rapporti di scambio e reciprocità. Tra gli espedienti tecnici menzionati, si è visto che nelle due sezioni la parola non è univoca e monosignificante, ma viene ampliata in un'utilizzazione sintattica che ne rende instabile anche il ruolo. La parola, già in queste prime prove, si libera dai legami che la radicano a un contesto grammaticale troppo vincolante, pur conservando lo spessore di un'origine umile, aderente alle cose, alla linearità di situazioni semplici e concrete.

\footnotetext{
49 Id., Magari poderse vestir, ivi, p. 11.

50 Testo ripreso da Ceserani R., Il testo poetico, Bologna, Il Mulino, 2005, p. 31.

«[...] quando la terra si muta in un'umida segreta / dove, timido pipistrello, la Speranza / sbatte le ali contro i muri e batte con la testa / nel soffitto marcito; [...] //» traduzione di Giovanni Raboni, ivi, p. 126.

51 Cergoly C. L., Sentà. La sigaretta, in Il portolano di Carolus, cit., p. 25.
} 


\section{BIBLIOGRAFIA}

BATTISTI, C., ALESSIO, G., 1968, Dizionario etimologico italiano, Vol. 1, G. Barbera Editore, Firenze.

BORSETTO, L., 1989, Lingua dialetto poesia. Cinque analisi: Marin, Cergoly, Calzavara, Giacomini, Bartolini, Edizioni Essegi, Ravenna.

BORSETTO, L., 1978, Trieste segreta. Le poesie mitteleuropee di Carolus L. Cergoly, in «La Rassegna della letteratura italiana», Anno $82^{\circ}$, n. 12, Sansoni, Firenze.

CERGOLY, C. L., 1938, Dentro de mi, Gabbiano, Trieste.

CERGOLY, C. L., 1943, Poesie a Barbara, Renato Fortuna, Trieste.

CERGOLY, C. L., 1970, Il portolano di Carolus. Poesie in dialetto triestino, Editrice La Galleria dei Rettori, Trieste.

CERGOLY, C. L., 1972, Canti clandestini. Nove poesie in lessico triestino, Tullio Misan libraio-editore, Trieste.

CERGOLY, C. L., 1973, Hohò Trieste. Ballatetta in lessico triestino, Editrice La Galleria dei Rettori, Trieste.

CERGOLY, C. L., 1976, Ponterosso. Poesie mitteleuropee in lessico triestino, «Quaderni della Fenice» n. 10, Guanda, Parma.

CERGOLY, C. L., 1980, Latitudine nord. Tutte le poesie mitteleuropee in lessico triestino, Mondadori, Milano.

CESERANI, R., 2005, Il testo poetico, Il Mulino, Bologna.

CHIESA, M., TESIO, G. (a cura di), 1978, Il dialetto da lingua della realtà a lingua della poesia, Paravia, Torino.

DAMIANI, R., 1973, Oggi, i poeti in dialetto, in «La Battana» A. X n. 30-31, Edit, Fiume-Rijeka.

DAMIANI, R., 1974, La miccia verde. Saggi di critica letteraria, LINT, Trieste.

DAMIANI, R., 1981, Poeti dialettali triestini. Profilo storico-critico (18751980), Lafanicola Edizioni «Italo Svevo», Trieste.

DAMIANI, R., GRISANCICH, C. (a cura di), 1989, La poesia in dialetto a Trieste, Edizioni «Italo Svevo», Trieste.

DEVOTO, G., OLI, G.C., 2007, Vocabolario della lingua italiana 2008, Le Monnier, Milano.

DORIA, M., 1991, Grande dizionario del dialetto triestino. Storico, etimologico, fraseologico, Edizione «Trieste Oggi», Trieste.

MAGRIS, C., 1982 (2009), Il mito absburgico nella letteratura austriaca moderna, Einaudi, Torino.

PANCRAZI, G., 1946, Scrittori d'oggi, Laterza, Bari.

PASOLINI, P. P., 1999, Carolus L. Cergoly, Inter pocula, poesie segrete triestine, in Saggi sulla letteratura e sull'arte, Tomo secondo (a cura di Siti W.-DE Laude S.), Mondadori, Milano.

PINGUENTINI, G., 1986, Nuovo dizionario del dialetto triestino. Storico, etimologico, fraseologico, Del Bianco, Modena. 


\section{POTRAGA ZA STILOM: PRVE ZBIRKE NA TRŠĆANSKOME NARJEČJU CAROLUSA L. CERGOLYJA}

Analiza ovoga rada temelji se na prvim zbirkama Carolusa L. Cergolyja napisanima na tršćanskome narječju Dentro de mi (Unutar mene) i Poesie a Barbara (Pjesme za Barbaru). U izabranim pjesmama definiraju se strukturalni temelji kroz pojedine stilske primjere koji omogućavaju definiranje Cergolyjeva lirskoga jezika kao pjesničkoga stvaralaštva. Tajje jezik neobično kreiranje kroz nove lingvističke susrete jer, dok otkriva djelovanje jezika, Cergoly stvara neobične spojeve, tako da dekonstrukcija i konstrukcija vrijede i kao svrhovit stvaralački čin negacije monovalentnosti riječi. Također Cergoly oblikuje jezik spajajući različiti jezični materijal, što dovodi do opsežnije sintaktičke i semantičke uporabe pjesničkoga izričaja.

Ključne riječi: Cergoly, Trst, pjesme, narječje.

\section{THE SEARCH FOR STYLE: THE FIRST OF CERGOLY'S COLLECTIONS IN TRIESTE DIALECT}

The analysis of this paper is based on the first collections of verse by Carolus $\mathrm{L}$. Cergoly written in the dialect of Trieste Dentro de mi (Inside Me) and Poesie a Barbara (Lyrics for Barbara). Through selected poems the structural foundation of these early Cergoly anthologies is defined with stylistic examples defining the poetic language. This language is a way to practice poetry in unusual patterns, because while revealing how language works, it causes unusual combinations, so that the deconstruction and construction are valid as operations essential to the denial of the unique word and monovalence. Also Cergoly shapes language combining various linguistic forms, leading to extensive syntactic and semantic use of poetic expression.

Key words: Cergoly, Trieste, poetry, dialect 